\title{
Coriolis Effect in Optics: Unified Geometric Phase and Spin-Hall Effect
}

\author{
Konstantin Y. Bliokh, ${ }^{1,2,3}$ Yuri Gorodetski, ${ }^{1}$ Vladimir Kleiner, ${ }^{1}$ and Erez Hasman ${ }^{1}$ \\ ${ }^{1}$ Micro and Nanooptics Laboratory, Faculty of Mechanical Engineering, and Russel Berrie Nanotechnology Institute, \\ Technion-Israel Institute of Technology, Haifa 32000, Israel \\ ${ }^{2}$ Institute of Radio Astronomy, 4 Krasnoznamyonnaya Street, Kharkov 61002, Ukraine \\ ${ }^{3}$ Nonlinear Physics Centre, Research School of Physical Sciences and Engineering, Australian National University, \\ Canberra ACT 0200, Australia
}

(Received 14 February 2008; revised manuscript received 15 May 2008; published 16 July 2008)

\begin{abstract}
We examine the spin-orbit coupling effects that appear when a wave carrying intrinsic angular momentum interacts with a medium. The Berry phase is shown to be a manifestation of the Coriolis effect in a noninertial reference frame attached to the wave. In the most general case, when both the direction of propagation and the state of the wave are varied, the phase is given by a simple expression that unifies the spin redirection Berry phase and the Pancharatnam-Berry phase. The theory is supported by the experiment demonstrating the spin-orbit coupling of electromagnetic waves via a surface plasmon nanostructure. The measurements verify the unified geometric phase, demonstrated by the observed polarization-dependent shift (spin-Hall effect) of the waves.
\end{abstract}

DOI: 10.1103/PhysRevLett.101.030404

Introduction. - The geometric phase is an inherent feature of the polarization optics that appears under evolution of electromagnetic waves in inhomogeneous and anisotropic media [1]. There are two types of geometric phases: (i) the spin redirection Rytov-Vladimirskii-Berry phase associated with the parallel transport of the wave field under $\mathrm{SO}(3)$ variations of the direction of propagation of the wave $[2,3]$ and (ii) the Pancharatnam-Berry phase that occurs under $\mathrm{SU}(2)$ manipulations with the polarization state of light $[4,5]$. In the general case, when both the direction of propagation and the polarization state of the wave are varied, the geometric phase becomes more intricate [6-9], and a unified geometrical description of the phase requires the tricky Majorana representation [8].

Another generalization of the geometric phases appears when one considers higher-order wave beams carrying an intrinsic orbital angular momentum (AM) accompanied by a phase vortex [10]. In contrast to spin AM (polarization), which equals $\sigma=1$, the orbital AM may take arbitrary integer values $\ell=0,1,2, \ldots$ (we determine AM per one photon in units of $\hbar$ ). The geometric and dynamic properties of light beams bearing orbital AM resemble the behavior of massless particles with spin $j=\sigma+\ell$. In particular, the spin redirection Berry phase and the Pancharatnam-Berry phase are generalized to the states of light with $\ell \neq 0[11,12]$. While the spin redirection phase is still associated with the parallel transport under $\mathrm{SO}(3)$ evolution [11], the Pancharatnam-type phase arises from $\mathrm{SU}(2 j)$ manipulation with the modes [12,13]. Accordingly, the Majorana formalism becomes drastically complicated for higher spins [14].

Whereas the Majorana representation develops the geometrical interpretation, the Berry phase can also be treated dynamically, as a manifestation of the spin-orbit interaction of waves carrying AM $[9,11,15,16]$. (In using the term "spin-orbit interaction" we refer to the interaction be-
PACS numbers: 03.65.Vf, 41.20.Jb, 42.15.-i, 42.25.-p

tween intrinsic $\mathrm{AM}$ and the extrinsic evolution of the wave.) In such an approach, the geometric phase turns out to be closely related to the Coriolis effect [17]. Furthermore, it has recently been shown that the spin redirection geometric phase is accompanied by the spinHall effect of light $[9,11,15,16,18]$, i.e., an AM-dependent displacement of the wave trajectory, which argues in favor of the dynamical interpretation of the Berry phase.

In this Letter, we develop and unify the dynamical approach to the geometric phases in optics. We show that, in the most general case, when a wave carrying an arbitrary AM changes its direction of propagation and state, the geometric phase is still given by a rather simple expression stemming from the Coriolis effect. The theoretical conclusions are confirmed experimentally using a focusing surface plasmon nanostructure. We observe that the geometric phases of partial waves bring about a polarization-dependent shift of the resulting intensity distribution which sheds light on the nature of the spin-Hall effect and explains other recent experiments. Our formalism can be equally applied to a number of problems outside the optical field. Indeed, the Coriolis effect is responsible for the Berry phase in classical [19] and quantum [20] systems, and the spin-Hall effect occurs in the evolution of various quantum particles [16,21].

Coriolis effect. -The total intrinsic AM of a paraxial wave equals $\mathbf{J}=J \mathbf{u}$, where $\mathbf{u}=\mathbf{k} / k$ is the vector indicating the direction of propagation of the wave ( $\mathbf{k}$ is the wave vector) and $J$ is the mean helicity of the wave which has a continuous spectrum in the range $[-j, j]$.

We consider a coherent noncollinear bundle of waves which are either (a) emitted or (b) scattered by a motionless body (Fig. 1). Let the observer measure the wave characteristics in a coordinate frame moving across the bundle and, hence, rotating with some instantaneous angular velocity $\boldsymbol{\Omega}$ (Fig. 1). Although the system is stationary in the labo- 
(a)

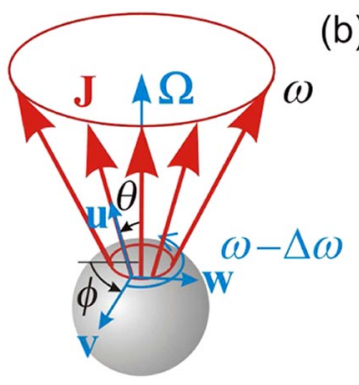

(b)

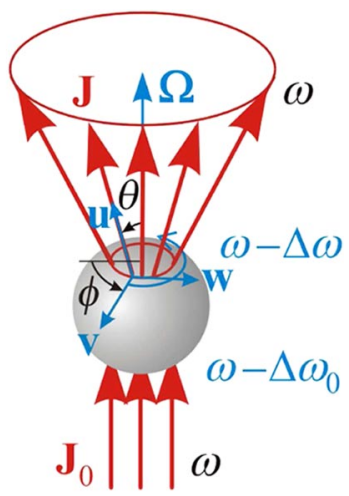

FIG. 1 (color online). The Coriolis effect observed in the rotating reference frame $(\mathbf{u}, \mathbf{v}, \mathbf{w})$.

ratory reference frame, the waves successively measured by the observer are related by the precession equation: $d \mathbf{u} / d t=\boldsymbol{\Omega} \times \mathbf{u}$. The frequencies of the waves measured in the rotating frame suffer the shift [case (a)] [22]

$$
\Delta \omega=-\mathbf{J} \cdot \mathbf{\Omega} .
$$

This shift corresponds to an analogous term in the wave Hamiltonian which is responsible for the spin-orbit interaction. In case (b), the observer will register the frequency difference between the input and output waves:

$$
\delta \omega=\Delta \omega-\Delta \omega_{0}=-\left(\mathbf{J}-\mathbf{J}_{0}\right) \cdot \boldsymbol{\Omega},
$$

where the subscript 0 indicates the characteristics of the input wave [Fig. 1(b)].

The frequency shift (1) is a manifestation of the Coriolis effect in the noninertial reference frame. It is identical to the frequency shift of the rotational modes of the Foucault pendulum staying on the rotating Earth $[\mathbf{J}$ is orthogonal to Earth's surface, and the angle $\theta$ between $\mathbf{J}$ and $\boldsymbol{\Omega}$ is determined by the latitude $\pi / 2-\theta$ (Fig. 1)] [19]. The shifts (1) and (2) are similar to the angular Doppler shifts (see [10]) that are produced as the medium moves with respect to observer with the angular velocity $-\boldsymbol{\Omega}$. However, unlike the real angular Doppler shift, there is no real rotation of the medium and energy exchange in our case. The Coriolis shifts (1) and (2) are artifacts of the rotation of the reference frame, and they indicate the inertia of the wave field.

Unified geometric phase.-The Berry phase for waves evolving in stationary media is a manifestation of the Coriolis effect. Indeed, the noninertial reference frame appears when the wave phase is measured in the coordinate frame attached to the varying direction of propagation $\mathbf{u}$ [17] (the case of the spin redirection phase) or to the polarization ellipse (the case of the Pancharatnam phase). In both cases, the geometric phase $\Phi=\int \Delta \omega d t$ originates from the Coriolis shift (1):

$$
\Phi=-\int \mathbf{J} \cdot \mathbf{\Omega} d t
$$

This phase is added to the wave phase calculated in a

parallel-transport (i.e., with $\boldsymbol{\Omega} \cdot \mathbf{J}=0$ ) reference frame (see Ref. [10(c)]). Although we considered the temporal evolution of the coordinate frame, Eq. (3) holds true for spatial evolution along a coordinate $\zeta$ as well. This case is described via the substitution

$$
d t \rightarrow d \zeta, \quad \mathbf{\Omega} \rightarrow \boldsymbol{\Omega}_{\zeta},
$$

where $\boldsymbol{\Omega}_{\zeta}$ is the rate of rotation of the coordinate frame with respect to the $\zeta$ coordinate.

First, when the wave in an AM eigenstate with integer $J=$ const propagates along a curved trajectory (so that the direction $\mathbf{u}$ is varied), and $\zeta$ is the coordinate along this trajectory, then the phase $\Phi$ is the spin redirection Berry phase [17]. If $\mathbf{u}$ is given by the spherical angles $(\theta, \phi)$ in the laboratory frame, then the attached reference frame $(\mathbf{v}, \mathbf{w}, \mathbf{u})$ (Fig. 1) suffers rotation with $\mathbf{J} \cdot \boldsymbol{\Omega}_{\zeta}=$ $J \cos \theta d \phi / d \zeta$, and Eqs. (3) and (4) result in

$$
\Phi=-J \int \cos \theta d \phi
$$

which is the known expression for the spin redirection Berry phase [1,3,11,17,23].

Second, let the wave now propagate in a fixed direction $\mathbf{u}=$ const, but the helicity $J$ is continuously varied with $\zeta$. This is accompanied by mixing of AM eigenstates and appearance of an oriented transverse structure of the wave (e.g., polarization ellipse). In the simplest case of $j=$ 1 , the state of the wave can be represented on the Poincaré (Bloch) sphere. In the spherical coordinates $(\vartheta, \varphi)$ on the Poincaré sphere, the wave helicity equals $J=\cos \vartheta$, whereas the angle of rotation of the polarization ellipse is $\varphi / 2$. The wave-accompanying coordinate frame $(\mathbf{v}, \mathbf{w}, \mathbf{u})$, with $\mathbf{v}$ attached to the polarization ellipse rotates with the angular velocity $\boldsymbol{\Omega}_{\zeta}=\mathbf{u}(1 / 2) d \varphi / d \zeta$, and Eqs. (3) and (4) yield

$$
\Phi=-\frac{1}{2} \int \cos \vartheta d \varphi,
$$

which is the known expression for the Pancharatnam-Berry phase $[1,5,23]$. For higher angular momenta $j>1$, the wave state is described by $\mathrm{SU}(2 j)$ structure $[12,13]$ and cannot be represented on the Poincaré sphere.

Finally, in the most general case of continuous evolution of a wave carrying an $\mathrm{AM} j$, with both the direction and the absolute value of $\mathbf{J}$ being varied, the higher-spin Majorana representation can be evoked. This advanced geometrical formalism represents the wave state by a set of $2 j$ vectors $\mathbf{n}_{i}, i=1, \ldots, 2 j$, on a unit sphere generalizing the $\mathbf{u}$ sphere of directions [14]. The geometric phase for cyclic evolutions is expressed by a rather complicated contour integral involving motion of these vectors on the sphere. Surprisingly, substitution of the rotational evolution $d \mathbf{n}_{i} / d \zeta=\boldsymbol{\Omega}_{\zeta} \times \mathbf{n}_{i}$ reduces this integral to the simple expression (3) for $\Phi$ [see Eq. (20) in Ref. [14]]. This expression remains valid for noncyclical evolutions provided the measurements are made in the reference frame 
attached to the direction of propagation and the transverse structure of the field.

Similarly, the phase difference due to Eq. (2),

$$
\delta \Phi=-\int\left(\mathbf{J}-\mathbf{J}_{0}\right) \cdot \mathbf{\Omega} d t,
$$

determines the geometric phases in systems with an abrupt transformation of the AM from $\mathbf{J}_{0}$ to $\mathbf{J}$ and a continuous evolution of $\mathbf{J}$. In this case, we imply that the incident wave is in an AM eigenstate and $\mathbf{J}_{0} \| \boldsymbol{\Omega}$. Otherwise, an additional geometric phase may appear due to motion of the $\mathbf{J}$-accompanying frame with respect to the incident wave (see below).

Experiment: Geometric phase producing the spin-Hall effect. - The experiment was performed utilizing surface plasmon-polariton waves and their strong polarization coupling sensitivity together with high surface confinement. The metallic sample (Fig. 2) has a semicircular corrugation (the inner radius is $1640 \mathrm{~nm}$ ) that acts as a lens for the surface plasmons $(\lambda=493 \mathrm{~nm})$ [24]. The grating was etched by a focused ion beam in a 100-nm-thick gold film evaporated onto a glass wafer. The coupling grating (period of $500 \mathrm{~nm}$ ) was accompanied by a Bragg grating (period of $250 \mathrm{~nm}$ ) from the outside. The grating grooves enclosed an azimuthal angle $\phi$ from 5 to 175 degrees in order to avoid resonant edge effects. The sample was illuminated from the bottom by a green laser ("Verdy" doubled Nd:Yag, $\lambda_{0}=532 \mathrm{~nm}$ ), while the near-field intensity of the surface plasmons was measured by a nearfield scanning optical microscope (Nanonics Multiview 2000) in a noncontact mode.

The scheme of the experiment involving wave vectors and polarizations is shown in Fig. 2. The incident light, propagating along the $z$ axis, is right- or left-hand circularly polarized: $\mathbf{J}_{0}= \pm \mathbf{e}_{z}$ (AM eigenstates). The coupling grating on the surface of the sample transforms the propagating waves into surface plasmons. They are linearly polarized (the electric field is normal to the metal surface), so that $\mathbf{J}=0$, and have the $\mathbf{k}$ vectors directed normally to the grating in the $(x, y)$ plane. Neither the spin redirection phase nor the Pancharatnam phase is applicable in this system, since the wave changes both its direction of propagation and polarization state. At the same time, the phase can be readily calculated using Eq. (7) with Eq. (4). The plasmon-accompanying coordinate frame $(\mathbf{v}, \mathbf{u}, \mathbf{w})$ is locally attached to the grating with $\mathbf{u}=\mathbf{k} / k$ and $\mathbf{v}=\mathbf{e}_{z}$. The azimuthal angle along the grating can be taken as the coordinate $\zeta$ in the above formalism, Fig. 2 [here the $(\mathbf{u}, \mathbf{v}, \mathbf{w})$ frame evolves with $\zeta$ across the bundle of plasmons, cf. Fig. 1]. In this manner, $\phi=\zeta, \mathbf{\Omega}_{\zeta}=\mathbf{e}_{z}$, and the phase difference appearing at the grating equals

$$
\delta \Phi=\int J_{0} d \phi= \pm \phi .
$$

Geometric phase (8) describes an azimuthal phase gradient in the plasmon field that depends on the polarization of the incident light. This changes the wave phase front

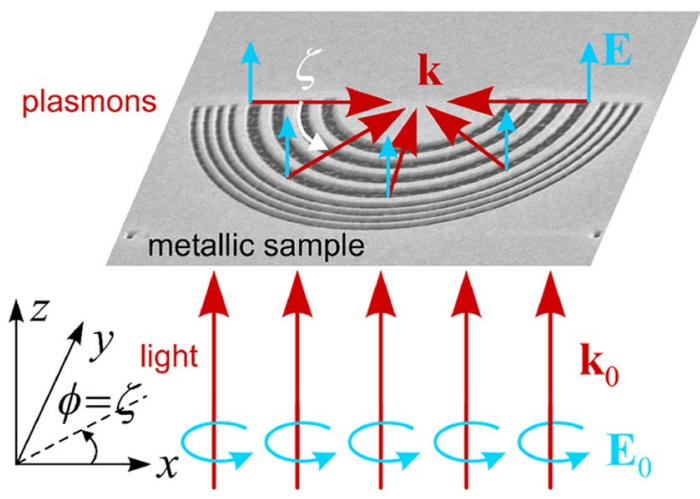

FIG. 2 (color online). Scheme of the experiment.

into a segment of spiral with the pitch $\lambda$ and the helicity dependent on the polarization of the incident wave (Fig. 3). As the surface plasmons are focused by the semicircular geometry of the sample, the phase (8) modifying the phase front brings about the transverse shift of the resulting focal spot. This shift can be estimated as

$$
\delta x \sim \pm \frac{\lambda}{2 \pi} .
$$

Since the shift (9) is proportional to the helicity of the incident wave $J_{0}= \pm 1$, it should be regarded as a spinHall effect of electromagnetic waves $[9,11,15,16,18]$. The spin-Hall effect originates from the polarization-dependent geometric phases (8) of partial waves forming the confined field in the focus. The polarization-dependent shift (9) is clearly seen both in experimental measurements and in numerical calculations processing the evolution of field with the phase (8) (Fig. 3), thereby confirming the geometric phase in the system.

Being a superposition of the right- and left-hand circular modes, the linearly $x$-polarized incident wave (which is not an AM eigenstate) brings about a split double spot in the output plasmon field (Fig. 3); cf. [24]. Such a field distribution is similar to a $\mathrm{HG}_{1,0}$ beam and has a phase singularity: a $\pi$ jump at $x=0$ (Fig. 3). This phase jump is a Panchratnam-type phase that arises due to a $\pi$ rotation of the polarization ellipse of the incident light with respect to the coordinate frame attached to the grating.

Discussion. - We have shown that the geometric phase is unified in terms of spin-orbit interaction and Coriolis effect and is given by a simple expression. Our experiment has verified the geometric phase in a rather complex system including light and surface plasmon polaritons. The results reveal the nature of the spin-Hall effect - the spindependent geometric phase of partial waves brings about a spin-dependent transverse shift of the resulting confined wave field. In contrast to semiclassical theories implying paraxial confined waves $[15,16,18,21]$, our experiment dealt with a highly nonparaxial field. As a result, the transverse shift is comparable with the focal spot width and is readily detected. 

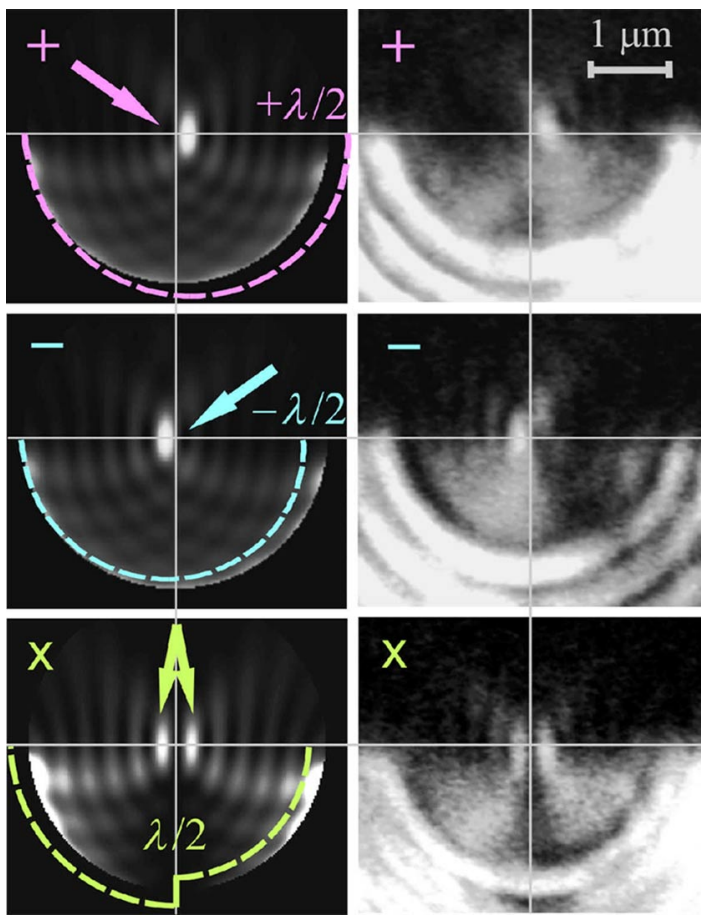

FIG. 3 (color online). Intensities of the plasmon field in the sample illuminated by the light of the right-hand $(+)$, left-hand $(-)$, and linear $x(X)$ polarizations. Left panels: Numerical calculations of the wave propagation with the phase fronts (dashed lines) disturbed by the geometric phase (8). Right panels: Experimental measurements.

Polarization-dependent displacements of light beams diffracted by a space-variant grating producing the Pancharatnam-Berry phase have been observed in Ref. [25]. A polarization-dependent transverse shift of the focal spot in lenses has also been reported in Refs. [26,27]. In Ref. [26], this effect was explained in the context of the spin-orbit interaction of photons in a vacuum. In a recent paper [27], deformation and shifts of the wave intensity in the focus of a high-numerical-aperture lens were treated as manifestations of the PancharatnamBerry phase and have been associated with the spin-Hall effect of light. Our approach can be directly applied to the systems in Refs. [24-27], clarifying the interrelations between the spin-orbit interaction, geometric phase, and spin-Hall effect of light.

The work by K.Y.B. is supported by the Linkage International Grant of the Australian Research Council.

[1] (a) S. I. Vinitskii et al., Sov. Phys. Usp. 33, 403 (1990); (b) Geometrical Phases in Physics, edited by A. Shapere and F. Wilczek (World Scientific, Singapore, 1989).

[2] S. M. Rytov, Dokl. Akad. Nauk SSSR 18, 263 (1938); V. V. Vladimirskii, ibid. 31, 222 (1941). See in Ref. [1(a)].

[3] J. N. Ross, Opt. Quantum Electron. 16, 455 (1984); R. Y. Chiao and Y.S. Wu, Phys. Rev. Lett. 57, 933 (1986); A.
Tomita and R. Y. Chiao, ibid. 57, 937 (1986); M. V. Berry, Nature (London) 326, 277 (1987).

[4] S. Pancharatnam, Proc. Indian Acad. Sci. A 44, 247 (1956). Reprinted in Ref. [1(b)].

[5] M. V. Berry, J. Mod. Opt. 34, 1401 (1987); R. Bhandari and J. Samuel, Phys. Rev. Lett. 60, 1211 (1988); R. Simon et al., ibid. 61, 19 (1988).

[6] R. Bhandari, Phys. Lett. A 135, 240 (1989).

[7] H. Jiao et al., Phys. Rev. A 39, 3475 (1989).

[8] J. H. Hannay, J. Mod. Opt. 45, 1001 (1998).

[9] K. Y. Bliokh, D. Y. Frolov, and Y. A. Kravtsov, Phys. Rev. A 75, 053821 (2007).

[10] (a) Optical Angular Momentum, edited by L. Allen, S. M. Barnett, and M.J. Padgett (Taylor \& Francis, London, 2003); (b) L. Allen, M. J. Padgett, and M. Babiker, Prog. Opt. 39, 291 (1999); (c) A. Bekshaev, M. Soskin, and M. Vasnetsov, arXiv:0801.2309.

[11] K. Y. Bliokh, Phys. Rev. Lett. 97, 043901 (2006); C. N. Alexeyev and M. A. Yavorsky, J. Opt. A Pure Appl. Opt. 8, 752 (2006); I. V. Kataevskaya and N. D. Kundikova, Quantum Electron. 25, 927 (1995).

[12] S. J. van Enk, Opt. Commun. 102, 59 (1993); M. J. Padgett and J. Courtial, Opt. Lett. 24, 430 (1999); E. J. Galvez et al., Phys. Rev. Lett. 90, 203901 (2003).

[13] G. S. Agarwal, J. Opt. Soc. Am. A 16, 2914 (1999); L. Allen, J. Courtial, and M. J. Padgett, Phys. Rev. E 60, 7497 (1999).

[14] J. H. Hannay, J. Phys. A 31, L53 (1998).

[15] V.S. Liberman and B. Y. Zel'dovich, Phys. Rev. A 46, 5199 (1992).

[16] A. Bérard and H. Mohrbach, Phys. Lett. A 352, 190 (2006).

[17] S. G. Lipson, Opt. Lett. 15, 154 (1990).

[18] K. Y. Bliokh and Y. P. Bliokh, Phys. Rev. E 70, 026605 (2004); Phys. Lett. A 333, 181 (2004); M. Onoda, S. Murakami, and N. Nagaosa, Phys. Rev. Lett. 93, 083901 (2004); K. Y. Bliokh and Y.P. Bliokh, ibid. 96, 073903 (2006); C. Duval, Z. Horváth, and P. A. Horváthy, Phys. Rev. D 74, 021701(R) (2006); O. Hosten and P. Kwiat, Science 319, 787 (2008).

[19] G. I. Opat, Am. J. Phys. 59, 822 (1991); A. Khein and D. F. Nelson, ibid. 61, 170 (1993).

[20] Y. A. Serebrennikov, Phys. Rev. B 73, 195317 (2006).

[21] G. Sundaram and Q. Niu, Phys. Rev. B 59, 14915 (1999); S. Murakami, N. Nagaosa, and S.-C. Zhang, Science 301, 1348 (2003).

[22] B. Mashhoon, Phys. Rev. Lett. 61, 2639 (1988).

[23] The phases (5) and (6) are determined in the coordinate frame rotated with azimuthal angles $\phi$ and $\varphi / 2$, respectively. Subtraction of this rotation leads to the substitution $-\cos \theta \rightarrow 1-\cos \theta$ and $-\cos \vartheta \rightarrow 1-\cos \vartheta$, and integrals in Eqs. (5) and (6) give the solid angles enclosed by the wave evolution on the corresponding spheres [1-5].

[24] L. Yin et al., Nano Lett. 5, 1399 (2005); F. Lopez-Tejeira et al., Nature Phys. 3, 324 (2007).

[25] Z. Bomzon et al. Opt. Lett. 27, 1141 (2002); E. Hasman et al., Prog. Opt. 47, 215 (2005).

[26] N. B. Baranova, A. Y. Savchenko, and B. Y. Zel'dovich, JETP Lett. 59, 232 (1994); B. Y. Zel'dovich, N. D. Kundikova, and L. F. Rogacheva, ibid. 59, 766 (1994).

[27] Z. Bomzon and M. Gu, Opt. Lett. 32, 3017 (2007). 